\title{
Associations between socio-economic status and body composition in an Irish maternal cohort
}

\author{
I. Coleman ${ }^{1}$, N. Kelly ${ }^{1}$, L. Mullaney ${ }^{1}$, A. O’Higgins ${ }^{2}$, M. J. Turner ${ }^{2}$ and D. McCartney ${ }^{1}$ \\ ${ }^{1}$ School of Biological Sciences, Dublin Institute of Technology, Kevin Street, Dublin 8, Republic of Ireland and ${ }^{2} U C D$ \\ Centre for Human Reproduction, The Coombe Women and Infants' University Hospital, Dublin 8, Republic of Ireland
}

Low socio-economic status (SES) has been identified as a significant predictor of poor dietary behaviours amongst young Irish women $^{(1)}$. These sub-optimal dietary behaviours are thought to contribute to adverse anthropometric status and poorer health outcomes among low SES women when compared with their more affluent peers ${ }^{(2)}$. Maternal obesity during gestation is a particular concern, as unfavourable body composition at this time has been shown to promote the development of obesity and chronic disease in the offspring ${ }^{(3)}$. This cross-sectional study aimed to investigate the relationship between low SES and sub-optimal anthropometric status in an Irish obstetric cohort.

Anthropometric data were collected using 8-lead, multi-frequency bio-electrical impedance analysis (BIA), while dietary data were simultaneously collected using the validated Willet food frequency questionnaire (WFFQ). All data were gathered at the initial maternal dating scan in the first trimester of pregnancy, at which time body composition remains unchanged ${ }^{(4)}$. Social class was divided according to occupation, following classifications set out by the central statistics office. Four hundred and three plausible dietary reporters were used in the final analyses. Independent samples t-tests and Mann Whitney $U$ tests were utilised, depending on the normality of distribution of the body composition parameters being tested.

\begin{tabular}{lccc}
\hline Physical Parameter & Low Social Class & High Social Class & Probability \\
\hline & Mean & Mean & $P$ Value \\
Body Mass Index $\left(\mathrm{Kg} / \mathrm{M}^{2}\right)^{*}$ & 25.00 & 24.1 & 0.08 \\
Fat Mass Index $\left(\mathrm{Kg} / \mathrm{M}^{2}\right)^{*}$ & 7.87 & 7.26 & 0.11 \\
Fat Free Mass Index $\left(\mathrm{Kg} / \mathrm{M}^{2}\right)^{*}$ & 17.20 & 16.8 & 0.04 \\
Body Fat Percentage $(\%)$ & 30.30 & 29.3 & 0.14 \\
Visceral Fat* & 3.89 & 3.35 & 0.03 \\
Waist Circumference $(\mathrm{cm})$ & 89.50 & 86.2 & 0.01 \\
Waist height Ratio* & 0.54 & 0.52 & 0.01
\end{tabular}

Social class was divided by occupation using classifications produced by the Central Statistics Office. Social Classes comprised the high social class group and classes 3 to 6 made up the low group. *Mann Whitney U Test used.

Social class had an inverse relationship with fat mass, particularly visceral fat mass, where adiposity is most strongly associated with adverse metabolic health outcomes. Unexpectedly, the fat free mass index of those in the lower social class grouping was significantly higher than that of their more affluent peers.

In conclusion, lower social class is associated with adverse body composition in the first trimester of pregnancy, most notably increased visceral adiposity. This may contribute to less favourable long-term health outcomes in the off-spring of these low-SES women, and represents an important intervention target to tackle socio-economic health inequalities ${ }^{(5)}$.

1. McCartney DM, Younger KM, Walsh J, O’Neill M, Sheridan C, Kearney JM (2013) Socio-economic differences in food group and nutrient intakes among young women in Ireland. Br J Nutr 110(11), 2084-97.

2. El-Sayed AM, Scarborough P, Galea S (2012) Unevenly distributed: a systematic review of the health literature about socioeconomic inequalities in adult obesity in the United Kingdom. BMC Public Health 12, 18.

3. Kent E, O'D wyer V, Fattah C, Farah N, O'Connor C, Turner MJ (2013) Correlation between birth weight and maternal body composition. Obstet Gynecol. 121(1), 46-50.

4. Fattah C, Farah N, Barry SC, O'Connor N, Stuart B, Turner MJ (2010) Maternal weight and body composition in the first trimester of pregnancy. Acta Obstet Gynecol Scand 89(7), 952-5.

5. McAvoy H, Sturley J, Sara B, Kevin B. Unequal At Birth. Inequalities in the occurrence of low birthweight babies in Ireland: The Institute of Public Health in Ireland; 2006. 